\title{
NOTES ON THE USE OF COMPATIBILITY INDEX IN THE ANALYTIC HIERARCHY PROCESS
}

\begin{abstract}
This study is composed of 3 notes associated with use and interpretation of the Compatibility [Similarity] Index (S.I.) in the AHP. Compatibility concerns how much two sets of priorities are mutually close. (A) a case is introduced to show that inconsistency in pairwise comparisons can detrimentally effect on measuring the compatibility between the two matrices. In order to avoid such errancy, we remind readers of the meaning of Saaty's compatibility metric and of correct usage. (B) originating from Saaty's consistency index for an entire hierarchy, compatibility for an entire hierarchy is derived to generalize the measurement of compatibility. (C) the sensitivity of S.I. is analyzed to figure out stable or unstable base.
\end{abstract}

Keywords: Compatibility Measurement, Compatibility for a Hierarchy, Sensitivity

\section{Introduction}

Saaty (1996) devised the compatibility [similarity] index (S.I.) by comparing two pairwise comparison matrices from the two priority vectors respectively. Using the consistency ratio, the standard values for the compatibility are also provided to determine whether two sets of judgments are compatible [close] or not. This is useful in validating or grouping the differences of multiple pairwise comparison matrices (Saaty \& Peniwati, 2008). S.I. can be used to consolidate conflicting assessors (Yoon \& Jung, 2005).

First, this paper shows correct meaning of S.I. and modifies S.I. not to lead to unexpected decision. Second, this paper derives compatibility for a hierarchy. The AHP is known by taking advantages of hierarchic decomposition (Saaty \& Vargas, 2012). Thereby, compatibility tests are required for an entire hierarchy. The third issue is related with the sensitivity of the S.I.. This paper discusses to improve the index.

\section{Compatibility [Similarity] Metric in the AHP}

Compatibility in the AHP is concerned with two different vectors from corresponding two matrices. Consistency is concerned with the compatibility of a matrix of the ratios constructed from a principal right eigenvector with the matrix of judgments from which it is derived. Let $W_{n x n}$ be the matrix of ratios of the principle right eigenvector $\left(w^{T}\right)$ of the positive reciprocal matrix $A_{n x n}$, and $\lambda_{\max }$ be the corresponding principal eigenvalue of $A$. Using the two matrices, $W$ and $A$, the Compatibility Index (S.I.) is defined as follows (Saaty 1996);

$$
\text { S. I. }=n^{-2} \cdot e^{T} A \circ W^{T} e=\lambda_{\max } / n
$$

where $\circ$ is the Hadamard product, that is, element-wise product of two matrices

International Symposium on the Analytic Hierarchy Process
WEB CONFERENCE DEC. 3 - DEC. 6, 2020 
ISAHP Article: A Style Guide for Paper Proposals To Be Submitted to the International Symposium on the Analytic Hierarchy Process 2020, Web Conference.

S.I. becomes 1 if and only if the two matrices are exactly same (i.e., matrix $A$ is perfectly consistent). Otherwise, S.I. goes beyond 1 . The right-hand side of (1) can be replaced by $1+C I(n-1) / n$, where $C I$ is consistency index. From the acceptance level of $C I$, we can derive the significance level of S.I. to assure that two matrices of ratio vectors are compatible (See Saaty 1996, p.63).

\section{Correct Use and Extension of Compatibility Measurement}

\subsection{Correct Use of S.I.}

Here is an example; Matrix A is given perfectly consistent $(\mathrm{CR}=0)$ and $\mathrm{B}$ is given with $\mathrm{CR}=0.10$, acceptable level. If we check S.I. by the equation (1), then $S . I_{A B}=1.073$, which is greater than the critical value of compatibility (1.067). It could be conclusion that two matrices are incompatible and therefore the two priority vectors are different.

However, the interpretation is not acceptable. Let's say that a standard matrix is composed of eigenvector elements derived from the given pairwise comparison matrix. If we compare the two standard matrices $(W, V)$ generated by the eigen vectors of the two given matrices, then $S . I \cdot W V=1.005$ which shows that two matrices are compatible or almost identical. This situation is from the fact that S.I. is twisted by some inconsistency.

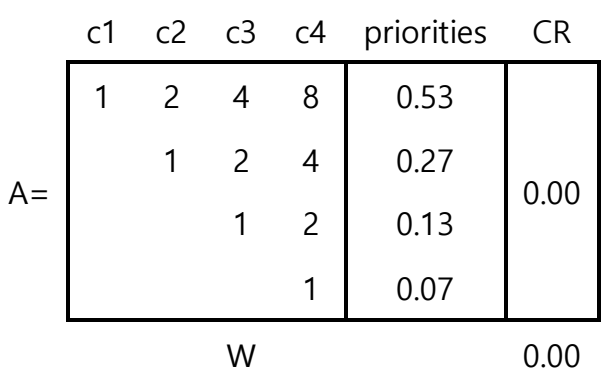

S.I._AB $=1.073>1.067$

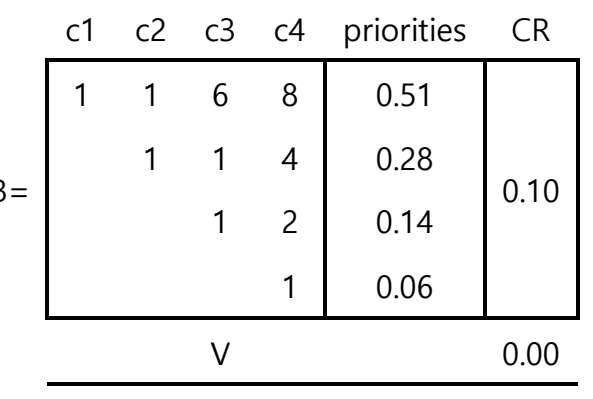

S.I._Wv $=1.005 \fallingdotseq 1.0$

\subsection{Compatibility Metric for an Entire Hierarchy}

We propose S.I. for an entire hierarchy $(\boldsymbol{S I H})$. The idea of SIH is based on the measurement of consistency ratio for an entire hierarchy $(C R H)$ (Saaty, 1980).

This paper semantically defines $\boldsymbol{S I H}$ as the combination of all S.I.s of pairwise comparison matrices with priorities of objects with respect to which the comparisons are made. Then, $S I H$ adds such results of combination in an entire hierarchy. However, considering consistency and its hierarchical priorities, what we define as $S I H$ is general form to aggregate S.I.s along layers of an entire hierarchy. Each S.I. is obtained by applying equation (2) with all factors in a layer. This is compared with the corresponding critical value of index $(\mathrm{SICH})$ obtained by taking acceptable levels of compatibility hierarchically given by Saaty(1996, p.63). Finally, the determination ratio of SIH and SIcH is DSIH as follows; 
ISAHP Article: A Style Guide for Paper Proposals To Be Submitted to the International Symposium on the Analytic Hierarchy Process 2020, Web Conference.

$$
\begin{gathered}
D S I H=\frac{S I H_{v w}}{S I c H_{v w}}=\frac{\sum_{h=1}^{H} S I_{v w}^{(h)}}{\sum_{h=1}^{H} S I C_{v w}^{(h)}}, \\
\text { where } \sum_{i=1}^{n_{h}} v_{i}^{(h)}=\sum_{i=1}^{n_{h}} w_{i}^{(h)}=1 \text { for } \forall h=1,2, \ldots, H
\end{gathered}
$$

$S I_{v w}^{(h)}:$ S.I. between two priorities vectors $v$ and $w$ in the $h$ th layer

$S I c_{v w}^{(h)}$ : Critical value of S.I. between two priorities vectors $v$ and $w$ in the $h$ th layer

$H$ : the number of layers with complete elements of a hierarchy below the goal

If $D S I H$ is equal or less than 1 , it can be determined that two sets of judgments for an entire hierarchy are not significantly different and categorized in a same group.

\subsection{Sensitivity Consideration}

S.I. measures differences of elements by ratios. However, if priority of element is smaller, the sensitivity even by small change is possibly higher. Concerning two vectors, there are a few ways to measure the difference between the two vectors according to interpretation perspective. S.I. is based on the pairwise comparison matrices derived by two eigenvectors. A simple alternative way is to measure the distances between two vectors. This study proposes Weighted S.I. (WSI), in which the weighted average is used.

\section{Concluding Remarks}

Researching the Compatibility in the AHP, this study focuses on the role of S.I. to determine whether two priorities vectors are similar or not. This paper shows that final S.I. is negatively affected by the inconsistency of pairwise comparisons. This paper has modified S.I. using the eigenvector and has proposed S.I. for an entire hierarchy $(S I H)$, and has discussed about sensitivity of S.I.. Empirical data analysis is required

\section{Key References}

Saaty, T.L. (1980). The Analytic Hierarchy Process. New York, McGraw-Hill.

Saaty, T.L. (1996). The Analytic Network Process. Pittsburgh, PA: RWS Publications.

Saaty, T.L., \& Peniwati, K. (2008). Group decision-making: Drawing out and reconciling differences. Pittsburgh, PA: RWS Publications.

Saaty, T.L., \& Vargas, L.G. (2012). Models, Methods, Concepts \& Applications of the Analytical Hierarchy Process. ${ }^{\text {nd }}$ Edition, Springer

Yoon, M.-S. \& Jung H.-W. (2005). A Study on the Utilization of Compatibility Metric in the AHP: Applying to Software Process Assessment, Proceedings of ISAHP in Hawaii. 